\title{
La mortalidad infantil como problema de derechos humanos: el caso venezolano
}

POR ANDY DELGADO BLANCO $\left.{ }^{*}\right)$

\begin{abstract}
Sumario: I. Introducción.- II. La mortalidad infantil como problema.- III. La mortalidad infantil y los derechos humanos.- IV. El caso venezolano.- V. A manera de cierre.- VI. Bibliografía.
\end{abstract}

Resumen: el propósito de este artículo es analizar la relación existente entre la mortalidad infantil y la vulneración de los derechos humanos de esta población por parte del Estado venezolano. Para cumplir con este objetivo se revisó el estado de la cuestión, los pactos e instrumentos internacionales que regulan los derechos humanos y que han sido suscriptos por la República, así como las disposiciones constitucionales y políticas en la materia. La revisión documental evidencia que en la presente centuria se interrumpió la tendencia al descenso de la mortalidad infantil, que venía desde la primera mitad del siglo pasado. Esto, además de mostrar la desigualdad social e inequidad en Venezuela, evidencia una regresión en materia de derechos y, más allá, una violación del derecho a la vida, a la salud y a la no discriminación.

Palabras claves: mortalidad infantil - derechos humanos - derecho a la salud - Venezuela

\section{La mortalité infantile liée au problème des droits de l'homme au vénézuéla}

Résumé: le propos de cette étude est d'analyser la relation entre la mortalité infantile et la baisse des droits des enfants au Vénézuela. Dans cet objectif, nous avons examiné les accords internationaux qui régulent les droits de l'Homme, accords signés par le gouvernement vénézuélien. Nous avons aussi examiné la Constitution et les programmes de Politique Publique. L'analyse de ces documents met en évidence qu'au cours de ce siècle, la tendance à la diminution de la mortalité infantile a disparu. Il s'agit là d' un indicateur de l'inégalité sociale au Vénézuela. Cette analyse met aussi en évidence que l'Etat Vénézuélien ne répond pas à son devoir de protéger

(*) Abogada. Jefa del Área Desarrollo y Salud del Centro de Estudios del Desarrollo (CENDES), Universidad Central de Venezuela. Dra. en Estudios del Desarrollo. Magister Scientiarum en Planificación del Desarrollo. Mención Política Social. 
les enfants. Il y a une régression des droits et une violation du droit à la vie, à la santé et à la non-discrimination.

\section{Mots clés: mortalité infantile - droits humaines - droit de la santé - Venezuela}

\section{Introducción}

La mortalidad infantil es un indicador de las condiciones de vida de la población y del respeto a los derechos humanos por parte de los Estados. Desde mediados del siglo pasado la tendencia de este índice ha sido hacia la baja, a nivel mundial, al punto que en los países de altos ingresos no se considera un problema de salud pública debido a la disminución de sus cifras. Pese a lo anterior, en África subsahariana y sur de Asia e incluso en algunos países de América Latina y el Caribe lo sigue siendo. Miles de niños siguen muriendo por causas evitables y prevenibles asociadas a la pobreza, deficientes condiciones de habitabilidad, carencia de agua potable y de sistema de excretas, entre otros factores.

Se trata de una situación de disparidad e inequidad social, donde la raza y la etnicidad son consideradas una desventaja; en mejores contextos y condiciones esta población tendría acceso a servicios de salud disponibles y de calidad, alimentos suficientes y un mejor hábitat. Los Estados son responsables del aumento - o no disminución - de la mortalidad infantil, cuando carecen de políticas públicas dirigidas a satisfacer de manera efectiva el derecho, a través de la mejora de las condiciones de salud de la población, y mediante la reducción de las brechas e inequidades existentes entre sectores sociales.

Entre otras características, los derechos humanos son universales e indivisibles; y cuando una parte de la población no tiene oportunidades iguales para disfrutar de ellos, cuando no hay atención oportuna y adecuada de la salud, ni se atienden los determinantes subyacentes a ella, o cuando no hay la posibilidad de disfrutar de los establecimientos, bienes y servicios necesarios para lograr el más alto nivel posible de salud o estos no están disponibles, ni son accesibles, aceptables y de buena calidad hay discriminación y, por tanto, una violación de sus principios básicos.

A mediados de la década de los años 40, Venezuela empezó a transitar el camino del campo a la ciudad, de la ruralidad a la urbanización, con mejoras en su panorama social, demográfico y político. Se formularon y ejecutaron políticas públicas de amplio espectro que, entre otros factores, incidieron positivamente en cambios sustantivos en el mapa de la salud de los venezolanos y muy especialmente de su población infantil. A partir de la primera década de esta centuria la situación ha ido cambiando, la tendencia a la disminución de la mortalidad infantil 
se revirtió y actualmente los índices de mortalidad infantil son unos de los más altos de la región.

En virtud de lo arriba señalado, este trabajo se propone analizar la vinculación existente entre mortalidad infantil y vulneración de derechos humanos en Venezuela. Para cumplir con este objetivo, los contenidos desarrollados se han organizado en tres partes: en la primera se discutirá lo que significa la mortalidad infantil, la situación a nivel mundial y en América Latina y el Caribe; en la segunda, se revisarán los tratados e instrumentos internacionales, en materia de derechos humanos relacionados con la infancia y, finalmente, a la luz de lo anterior, se analizará la situación en Venezuela.

\section{La mortalidad infantil como problema}

El desarrollo está ligado a las oportunidades que tienen las personas para vivir con dignidad y calidad a lo largo de su vida. Durante las primeras etapas de su desarrollo evolutivo el ser humano es especialmente vulnerable; los niños dependen de los adultos de su entorno familiar y social para que sus necesidades sean satisfechas, lo que obliga a la sociedad, y dentro de esta al Estado y la familia, a crear un mínimo de condiciones, formales y materiales, para lograrlo, entre las que destacan por ser objeto del tema objeto de estudio, las vinculadas a la salud.

En lo formal, la garantía del derecho a la vida y a la salud; en lo material, el diseño y ejecución de políticas públicas que impacten positivamente en el bienestar de la población infantil, con énfasis en el acceso a cuidados en salud, nutrición y educación dirigidos a la madre y al niño suelen ser indispensables, así como los recursos suficientes para hacerlos efectivos. Cuando no se alcanzan mínimos satisfactorios en estas materias, la subsistencia se ve comprometida y la vida llega a su fin en las etapas tempranas del crecimiento, por causas evitables y prevenibles.

La mortalidad infantil como indicador de salud refleja las condiciones de vida de la población y la situación sanitaria, ergo, el grado de desarrollo de un país. La relación entre acceso oportuno a servicios de salud de calidad es directamente proporcional a las oportunidades de la infancia para disfrutar del derecho a la vida. Mientras más alta es la tasa de mortalidad, más baja es la cobertura y calidad de los servicios; la erradicación de enfermedades, los cuidados prenatales, las condiciones de higiene (saneamiento básico y suministro de agua potable, por ejemplo), la cantidad de centros de salud y su equipamiento por número de habitantes, la disponibilidad e ingesta de alimentos adecuados e incluso el grado educativo de la madre son factores que favorecen la reducción de la tasa de mortalidad infantil. 
La mortalidad infantil se refiere al número de niños menores de un año de edad fallecidos a lo largo de un periodo determinado. La tasa de mortalidad infantil expresa la proporción existente entre el número de niños menores de un año fallecidos en el curso de un año y el número de nacimientos vivos durante el mismo año. Tiene varias categorías: a) neonatal precoz, que abarca desde el nacimiento hasta la primera semana de vida; b) neonatal, hasta el primer mes de vida; y c) postneonatal, desde el nacimiento hasta un año de edad. Cabe destacar que, en algunos casos, esos índices abarcan a niños mayores de un año hasta los cinco o nueve años y se llama infantil (Mortalidad Infantil, s/f). De acuerdo con Pison, el $40 \%$ de todas las defunciones de menores de cinco años ocurren durante el período neonatal, esto es, en el primer mes de vida; el $26 \%$ de estas muertes - que representan el $10 \%$ de todas las defunciones de niños menores de cinco años- son producidas por infecciones graves, originadas por malas condiciones del embarazo, complicaciones durante el parto, sepsis neonatal, neumonía, diarrea, paludismo o por enfermedades infecciosas que pudieron tratarse o prevenirse (OPS, 2001; Pison, 2010).

Investigaciones en la materia evidencian que la pobreza tiene una relación significativa con ciertas condiciones y enfermedades asociadas a la mortalidad infantil, como la desnutrición, diarreas, enfermedades respiratorias agudas, las de origen infeccioso, entre otras. Bolsi et al (2009) han definido los riesgos de enfermarse y morir durante el primer año de vida a través de dos niveles de condicionantes: lo macro-institucional y lo micro-institucional. El primero lo constituye la estructura socioeconómica de un país, en la que distingue factores asociados a la accesibilidad a los servicios públicos, organización de la producción, patrones de distribución de los ingresos y beneficios sociales, distribución y costo relativo de servicios; la producción de alimentos, deficiencias alimenticias, existencia de vectores de enfermedades transmisibles; formas de organización, gestión, políticas y eficiencia del sistema formal de salud. El segundo, está relacionado con el contexto familiar, nivel de instrucción de los padres, capacidad económica, calidad de la vivienda, edad de la madre, condiciones de habitabilidad, dieta, por solo mencionar algunos de ellos (Augsburger et al, 2006; Dhrifi, 2018).

Sin negar lo indicado en el párrafo anterior, es menester destacar que la pobreza o un entorno económico con cifras negativas no son suficientes para explicar altas tasas de mortalidad infantil. Diversos estudios (Díaz, 2003; Aguirre, 2009, entre otros) han mostrado que una actuación gubernamental oportuna, a través de políticas sanitarias preventivas, puede ser significativa para revertir o disminuir los efectos perversos de la pobreza en la salud infantil. Las enfermedades inmunoprevenibles pueden disminuirse con la aplicación de las dosis requeridas de vacunas contra tuberculosis, poliomielitis, difteria, tosferina, tétanos y sarampión. Las diarreicas, con medidas de saneamiento ambiental e inversiones en infraestructura, 
para que la población cuente con agua potable y redes de alcantarillado, o si los centros de atención de salud contasen con terapias de hidratación oral; en el caso de las infecciones respiratorias agudas probablemente bastaría con su adecuada referencia a los centros de salud o, en general, con contar con una infraestructura hospitalaria y recursos humanos capacitados para brindar una adecuada atención prenatal y médica durante el parto, tecnología avanzada, como las imágenes de ultrasonido para mejorar la labor diagnóstica.

Desde el siglo pasado y hasta las dos primeras décadas de la presente centuria, en la medida en que las condiciones de vida de la población fueron mejorando, se contó con servicios de agua potable y saneamiento, avances en la medicina preventiva y en el tratamiento o erradicación de enfermedades como diarrea, paludismo, sarampión, neumonía, viruela y tuberculosis la mortalidad de los niños menores de cinco años comenzó a descender de manera sostenida en el mundo. En los treinta años que van de 1960 a 1990 hubo una reducción de un 60\% de la tasa de mortalidad en la infancia, cuando murieron 12,6 millones de niños. Para 2006, por primera vez, la cifra total de muertes anuales entre los niños menores de cinco años fue inferior a los 10 millones, 72 muertes por cada 1000 nacidos vivos, un 23\% menos que en 1990; en 2017 fue de 5,4 millones. Estas reducciones han sido sobre todo significativas en los países de ingresos altos, que presentan tasas muy bajas, al punto que, para 2017, la proporción era de 1 de cada 185 niños. En 2019 el número total de defunciones de niños menores de cinco años disminuyó a 5,2 millones, la tasa mundial de mortalidad se redujo en un 59\%, y pasó de 93 defunciones por 1000 nacidos vivos en 1990 a 38 defunciones por 1000 nacidos vivos en 2019; mientras que en 1990 uno de cada 11 niños moría antes de los cinco años, en 2019 la proporción fue de uno de cada 27 (GIEMN, 2018; Orbea, 2019; OMS, 2020).

A diferencia de lo ocurrido en los países desarrollados, en los subdesarrollados los progresos han sido más lentos y menos promisorios. Un bebé nacido en África subsahariana o en Asia meridional tiene nueve veces más probabilidades de morir durante su primer mes de vida que uno nacido en un país de altos ingresos. En 2017, la mitad de las muertes de niños menores de 5 años se registraron en el África subsahariana, donde uno de cada 13 niños murió antes de alcanzar los cinco años y el 30\%, en Asia meridional, en la que 2,5 millones de recién nacidos murieron durante el primer mes, lo que pudiera explicarse por los déficits y bajas posibilidades de acceso que tienen las personas a servicios sociales básicos, como educación, salud, agua potable, nutrición adecuada, higiene y saneamiento (Dhrifi, 2018).

Las disparidades antes señaladas también son observables en un mismo país; diversas investigaciones muestran que en las zonas rurales las tasas de mortalidad 
de menores de cinco años son, como media, un 50\% más altas que en las zonas urbanas. Además, los nacidos de madres sin formación tienen más del doble de probabilidades de morir antes de cumplir los cinco años que aquellos cuyas madres han realizado estudios secundarios o superiores (GIEMN, 2018).

En América Latina y el Caribe, una región de 630 millones de personas, de los cuales 193 millones son niños y adolescentes, la tendencia es mucho más alentadora, en términos generales, y, al mismo tiempo, en lo específico, indicadora de las desigualdades e inequidades que marcan la región. Desde 1960 hasta la actualidad, la tasa de mortalidad infantil ha venido descendiendo de manera significativa. A inicios de los sesenta se contabilizaban 102 niños fallecidos por mil nacidos vivos; en 1970, aproximadamente 1 de cada 12 moría antes de cumplir el primer año de vida y 1 de cada 9 antes de los cinco años. Desde 1990, las muertes infantiles de menores de 5 años se han reducido en un $71 \%$. En ese año, murieron 652 niños, 1 de cada 25 niños no llegaba a cumplir el primer año de vida y 1 de cada 20 moría antes de los cinco años; entre el 2000 y el 2017, la tasa promedio de mortalidad infantil disminuyó en un 35\% y la mayoría de los países registró descensos entre el $25 \%$ y el $45 \%$. Para 2010, 1 de 45 niños murió antes de cumplir un año y 1 de 36 antes de los cinco; siete años después, en 2017, 187 mil menores de cinco años morían cada año, uno cada tres minutos y el $52 \%$ antes de cumplir 28 días de nacidos. El promedio de mortalidad infantil era de 15,7 muertes por cada 1.000 nacidos vivos. Actualmente, un tercio de las muertes infantiles reportadas en la región, durante el primer año de vida, ocurren en el período neonatal - primeras cuatro semanas de vida o desde los días 0 al 27-(OMS, 2014; Cepal y Unicef, 2011; Unicef, 2018 y Aguirre, 2009).

Las cifras que se vienen comentando aumentan según de que países se trate o las comunidades, etnia o raza de procedencia de esos niños; bien sean rurales, indígenas y afrodescendientes, quienes presentan una "sistemática sobre-mortalidad" respecto del resto de la población (Cepal, 2010, p. 5) e incluso dependiendo del nivel de nivel de ingresos familiar y nivel educativo de la madre. Cabe destacar que la ubicación geográfica (urbana o rural), aun cuando constituye un determinante de la mortalidad infantil en la región, no llega a tener el mismo peso que los ingresos o la escolaridad de la madre.

Para 2017, en países como Cuba, Antigua y Barbuda, Bahamas y Chile la mortalidad fue menor (menos de 7 muertes por cada 1.000 nacidos vivos), en tanto que en Guyana, Bolivia y sobre todo Haití fue mayor (26, 28 y 54 por cada 1.000 nacidos vivos, respectivamente). Antigua y Barbuda, Bahamas, Brasil y Perú registraron caídas superiores al 55\%; mientras que Granada y Venezuela experimentaron aumentos en la tasa de mortalidad infantil, en particular esta última, con un incremento de casi el 40\%. En Colombia, la mortalidad infantil es más de 4 veces 
mayor en el quintil más pobre que en el quintil más rico, y casi 5 veces mayor cuando las madres tienen un escaso nivel de escolaridad que cuando tienen un nivel superior. En las zonas rurales del Perú, la tasa de mortalidad infantil llega a 25 muertes por cada 1.000 nacidos vivos, en comparación con 16 muertes por cada 1.000 nacidos vivos que se registran en las zonas urbanas (OCDE, 2020).

Especial atención merece el determinante étnico en la región. De acuerdo con la Cepal (2010), en el primer quinquenio del 2000, la mortalidad infantil de los niños indígenas fue un $60 \%$ mayor que la de no indígenas ( 48 por mil nacidos vivos frente a 30 por mil, respectivamente), con una probabilidad de morir antes de los cinco años de vida. Brecha que aumentaba con una sobremortalidad del $70 \%$ cuando pertenecían a los pueblos Campa Ashaninka y Machiguenga del Perú, Wayú de Venezuela, Tzoztil de México y Xavante en Brasil. Los riesgos más altos se encontraban en Panamá, Ecuador, Venezuela y Paraguay. En el primero, un niño indígena tenía tres veces más probabilidades de fallecer antes de cumplir un año que un niño no indígena, riesgo que se incrementaba para los menores de cinco años. En los otros tres países, el riesgo de muerte es el doble para los indígenas tanto para la mortalidad infantil como en la niñez.

Pese a los notables avances en la materia, en lo cualitativo y cuantitativo, los datos arriba mostrados son indicativos de un problema prioritario en materia de salud; y, más allá de eso, evidencian una flagrante violación a los derechos humanos de un significativo contingente de la población, como se detallará en las líneas que siguen.

\section{La mortalidad infantil y los derechos humanos}

Conforme se desprende de la Declaración Universal de los Derechos Humanos, proclamada por la Asamblea General de las Naciones Unidas en París, el 10 de diciembre de 1948, el desiderátum de los Derechos Humanos es el reconocimiento universal de la dignidad humana independientemente de la raza, color, sexo, idioma, religión, opinión, origen nacional o social, posición económica, nacimiento o cualquier otra condición. Dignidad que, entre otros aspectos, está íntimamente vinculada con un nivel de vida adecuado y mínimos de salud, bienestar y asistencia, los cuales, a tenor de lo dispuesto en el artículo 25 de la Declaración, deben brindarse de manera especial a los niños a fin de garantizarles una vida plena, larga y saludable (Naciones Unidas, 1948).

Las muertes infantiles, prematuras y prevenibles, obedecen a desigualdades en la atención médico-sanitaria y carencias sociales estructurales que podrían evitarse o al menos disminuirse a través de intervenciones orientadas a influir sobre los determinantes sociales y económicos, que dan origen a las inequidades en salud. 
Diversas investigaciones en la materia (Medina Gómez y López Arellano, 2011; Abriata y Fandiño, 2010; Augsburger, 2006, entre otros) han evidenciado que los niños que habitan en zonas rurales o entornos residenciales precarios pertenecen a hogares de bajos ingresos o pobres, cuyas madres tienen menores niveles educativos, embarazos tempranos no deseados; habitan en países con bajos niveles de gasto social en salud, insuficiente provisión de servicios públicos, soporte nutricional, con deficiencias en seguridad social y carencias de protección estatal y "tienen una mayor probabilidad de experimentar problemas de desarrollo y de salud desde que nacen y de acumular riesgos sanitarios durante el crecimiento" (Dhrifi, 2018 p. 73), por lo que pasan a formar parte de las estadísticas en las cuales la mortalidad infantil muestra una alta recurrencia.

La mortalidad infantil es un problema de derechos humanos desde el momento en que nacer o vivir en ciertos hogares, zonas geográficas o residenciales está asociado a un mayor riesgo de padecer enfermedades, que constituyen una alta probabilidad de muerte o cuando un niño no tiene acceso a atención oportuna y de calidad cuando nace (o a su madre en el embarazo, parto o puerperio) o en el transcurso de su desarrollo evolutivo; y lo es porque esas circunstancias no permiten la garantía y realización efectiva de los derechos a la vida, la integridad y la salud, lo que constituye una violación flagrante de los derechos humanos; porque esas muertes no hubieran ocurrido si esos niños o sus familias hubiesen tenido acceso oportuno a una atención sanitaria de calidad, con tecnología médica, o no hubieran sido excluidos o se les hubiera condicionado ese acceso en función de su clase socio económica, condición de ruralidad, etnicidad o raza. Quienes fallecen en estos casos son hijos de familias pobres, de minorías étnicas o raciales, de clases sociales bajas, que viven en zonas rurales aisladas o en zonas urbanas marginadas.

La suma de brechas y disparidades aunada a la carencia de políticas públicas dirigidas a promover la justicia social, la equidad e igualdad, entre otras razones, ha condicionado la reflexión sobre la necesidad de ofrecer oportunidades a las personas, a través de compromisos individuales y sociales. En el marco de estos últimos, con el propósito de poner fin a las discriminaciones de las personas y salvaguardar sus derechos, muy especialmente los de la población infantil, desde el siglo pasado la comunidad internacional ha dado pasos progresivos en aras de garantizar sus derechos y eliminar —o disminuir, según sea el caso- las desigualdades e inequidades, a través de diferentes instrumentos jurídicos, como declaraciones, acuerdos, conferencias y resoluciones, entre las destacan, en orden cronológico, por su relación con el tema que se viene exponiendo, los siguientes:

a) Declaración de los Derechos Humanos, firmada en 1948, en la que se enfatiza en el derecho a cuidados y asistencia especial de la maternidad y la infancia. 
b) Convención sobre los Derechos del Niño de 1989, ratificada por 194 Estados, los cuales reconocen que: a) todo niño tiene el derecho intrínseco a la vida; b) se comprometen a garantizar en la máxima medida posible su supervivencia y desarrollo, mediante derechos como: el más alto nivel posible de salud, acceso a servicios para el tratamiento de las enfermedades y la rehabilitación de la salud, y que ningún niño sea privado del disfrute de los servicios sanitarios y a tomar medidas para reducir la mortalidad infantil y en la niñez.

c) Pacto Internacional de Derechos Económicos Sociales y Culturales (PIDESC), de 1966, que establece a los Estados Parte la obligación de adoptar medidas especiales de protección y asistencia en favor de todos los niños y adolescentes, con miras a reducir su mortinatalidad, mortalidad y a fomentar su sano desarrollo.

d) Cumbre Mundial en Favor de la Infancia de 1990, entre cuyos objetivos estaba mejorar la vida de la niñez y las mujeres en el plazo de un decenio, para lo cual se adoptó un Plan de Acción, entre cuyas metas destacaba la reducción de la mortalidad neonatal, infantil y materna y el monitoreo de las muertes infantiles, lo que incidió en avances notables de los sistemas de recolección de información y procesamiento de datos sobre la situación de la infancia que permitieron conocer de fuentes confiables el aumento o disminución de las tasas de mortalidad infantil en regiones y países (Naciones Unidas, 1990).

e) Observación General No 14 formulada en el año 2000 por el Comité de Derechos Económicos, Sociales y Culturales, órgano encargado de supervisar la aplicación del PIDESC, en la que se establece el contenido normativo del Derecho a la salud, se fijan los elementos esenciales para evaluar su cumplimiento y se insta a los Estados a adoptar medidas y recursos que contribuyan a mejorar la salud infantil, reducir la mortinatalidad y la mortalidad infantil y promover el sano desarrollo de los niños. Cabe destacar la mención a que el interés superior del niño y el adolescente será "primordial" en los programas y políticas que garanticen el derecho a la salud de esta población su no discriminación, y derecho al acceso a una alimentación adecuada, un entorno seguro y servicios de salud física y mental.

f) Cumbre del Milenio, celebrada en el año 2000, en la que se fija reducir la mortalidad de los niños menores de cinco años en dos terceras partes para 2015. En ese marco la mortalidad infantil se redujo a "casi la mitad, debido a una mejor nutrición, atención de salud y calidad de vida"; pero las tasas de mortalidad de los menores de 5 años siguieron siendo "inaceptablemente 
altas, en especial considerando que la mayoría de los decesos son por causas evitables o tratables" (BM, 2015, p. 1).

g) Sesión Especial en favor de la Infancia de la Asamblea General de la Organización de las Naciones Unidas celebrada en 2002. El resultado fue un documento llamado Un Mundo Apropiado para los Niños y las Niñas en el que los gobiernos prometen mejorar las vidas de la población infantil en un plazo de diez años, con los recursos necesarios; en materia de salud los compromisos fueron: medicinas vitales a precios asequibles y tratamiento y promoción de una salud mejor.

h) En septiembre de 2012, durante la 28 ${ }^{\text {a }}$ Conferencia Sanitaria Panamericana de la Organización Panamericana de la Salud (OPS), las autoridades sanitarias de las Américas aprobaron una estrategia y plan de acción para la salud integral en la niñez. Este Plan está dirigido a mejorar la salud y reducir la mortalidad infantil a través de un enfoque sanitario integral y multisectorial basado en los derechos y los determinantes sociales de la salud. Las estrategias diseñadas se centran en la atención primaria de la salud, con un enfoque de género, intercultural.

i) Agenda 2030 para el Desarrollo Sostenible, con sus Objetivos de Desarrollo Sostenible. En septiembre de 2015 las Naciones Unidas fijaron nuevos compromisos y metas, esta vez para el 2030. Entre ellas se cuentan poner fin a las muertes evitables de recién nacidos (al menos a 12 por cada 1000 nacidos vivos en cada país) y de niños menores de 5 años (al menos a 25 por cada 1000 nacidos vivos en cada país). Esto significa reducir la tasa mundial de mortalidad materna a menos de 70 defunciones por 100000 nacidos vivos, y poner fin a todas las formas de malnutrición, una causa frecuente de defunción de niños menores de cinco años.

Revisar el cumplimiento del derecho a la salud, desde la óptica de los derechos humanos, pasa por examinar la forma en que un Estado honra los compromisos que ha asumido para respetar, proteger y garantizar su efectivo ejercicio; sus avances o retrocesos, formales y materiales, y, las oportunidades que tienen las personas para su disfrute efectivo. En términos del derecho a la salud, a tenor de lo dispuesto en la Observación General 14 (OG14) los Estados tienen la obligación de respetar el derecho absteniéndose de denegar o limitar el acceso igual de todas las personas a los servicios de salud preventivos, curativos y paliativos; lo que incluye abstenerse de imponer prácticas discriminatorias como política de Estado. La obligación de proteger incluye el adoptar leyes u otras medidas para velar por el acceso igual a la atención de la salud. 
La obligación de cumplir requiere el reconocimiento del derecho en el sistema político y en el ordenamiento jurídico nacional, mediante la aplicación de leyes, y la adopción de una política nacional de salud acompañada de un plan detallado para el ejercicio del derecho a la salud. A estos efectos la OG14 (2000) específica que los Estados "deben garantizar la atención de la salud, en particular estableciendo programas de inmunización contra las principales enfermedades infecciosas, y velar por el acceso igual de todos a los factores determinantes básicos de la salud, como alimentos nutritivos sanos y agua potable, servicios básicos de saneamiento y vivienda y condiciones de vida adecuadas".

La obligación de facilitar requiere en particular que los Estados adopten medidas positivas que permitan y ayuden a los particulares y las comunidades disfrutar del derecho a la salud. Comprende la obligación de facilitar, proporcionar y promover. Los Estados Partes también tienen la obligación de facilitar el derecho a la salud en los casos en que los particulares o los grupos no están en condiciones, por razones ajenas a su voluntad, de ejercer por sí mismos ese derecho con ayuda de los medios a su disposición. La obligación de promover el derecho requiere que los Estados emprendan actividades para promover, mantener y restablecer la salud de la población.

Los instrumentos, convenciones y acuerdos internacionales arriba mencionados son significativos, pero no suficientes. La sola titularidad del derecho no basta y, para decirlo en términos de Amartya Sen (2000 y 2002), se requiere de oportunidades para obtener ciertos mínimos sociales que las personas valoren o tengan razones para valorar. Cuando las estadísticas muestran un aumento de la mortalidad infantil debido al repunte o a la aparición de enfermedades perfectamente evitables mediante políticas de salud eficientes y universales, lo que verdaderamente está de fondo es la ausencia de ciertas libertades reales y las limitaciones existentes en el goce de la igualdad de estas libertades.

De lo arriba señalado se desprende la importancia de interrogarse sobre las oportunidades con las que cuenta la población infantil para vivir en la sociedad en la que se desenvuelve y cuáles las capacidades - el poder- que posee para evitar enfermedades o una muerte prematura, y así sopesar las desigualdades que existen en el interior de la sociedad. Una respuesta a la interrogante anterior se encuentra en la articulación del contenido del derecho a la salud con la posibilidad de exigir su realización progresiva mediante políticas públicas específicas, en tiempo y espacio, que vayan más allá de atender necesidades de la infancia y la familia y se dirijan a la satisfacción del derecho.

Las altas tasas de mortalidad infantil evidencian una violación de los derechos humanos de esta población. Los Estados, en tanto titulares de las obligaciones 
derivadas de los derechos a la igualdad, la vida, la no discriminación y la salud, por solo citar algunos, son responsables de garantizarlos de manera efectiva.

Como corolario de lo anterior cabe destacar que un Estado es responsable del aumento -o no disminución-de la mortalidad infantil, cuando carece de políticas públicas dirigidas a satisfacer el derecho, a través de la mejora de las condiciones de salud de la población, y mediante la reducción de las brechas e inequidades existentes entre sectores sociales.

\section{El caso venezolano}

A principios del siglo pasado, Venezuela era un país rural, sin un sistema interconectado de carreteras ni de sanidad pública, con una población diezmada por enfermedades como el paludismo y la tuberculosis. A partir de 1936, con el "Programa de Febrero", impulsado por el General López Contreras, la acción estatal, a través del recién creado Ministerio de Sanidad y Asistencia social, se dirigió a resolver las carencias de la población en materia de sanidad e higiene pública, para lo cual se empezó a formar una red institucional de apoyo. Para ese momento la mortalidad infantil era de 121,7 por 1000 nacidos vivos y en algunas entidades federales superaba los 200 (Yánez, 2013 y Páez, 2018).

En la década del cuarenta se creó una red asistencial a la cual tuvo acceso gran parte de la población, aunque de manera desigual, en cuanto a cobertura y calidad. Los sanitaristas de la época adoptaron una concepción integral de la sanidad pública dirigida a erradicar las enfermedades endémicas, diseñaron y desarrollaron campañas de vacunación, programas de puericultura y atención prenatal, por solo citar algunos. El Estado dispuso recursos para aliviar y tratar las enfermedades, aumentó el consumo calórico, lo que influyó en la mejora de las condiciones de vida y salud de la población. La tasa de mortalidad infantil inició una tendencia a la baja, hasta llegar a 80 por 1000 nacidos vivos registrados hacia el final de esa década (Castellanos, 1982 y Yánez, 2013).

Al cierre de los setenta y sobre todo en los ochenta, en medio del crecimiento demográfico, desajustes socioeconómicos, mal manejo de los recursos del Estado y clientelismo político, la crisis económica que azotaba a la región impactó a lo interno y las condiciones de vida de la mayoría de la población desmejoraron. En materia de salud, el modelo institucional se limitó a las actividades curativas e individuales. Aun así, siempre según Yánez (2013), las tasas de mortalidad infantil siguieron descendiendo de 32 a 24 por 1000 nacidos vivos registrados en los noventa.

En esa última década del siglo pasado, Venezuela vivió una convulsa situación política, social y económica expresada en insurrecciones al orden constitucional, renuncia de un presidente de la República, baja de los precios del petróleo, 
inflación y desabastecimiento. El agotamiento del modelo de desarrollo incidió en que en 1999 se convocase la última Asamblea Constituyente del siglo XX con el fin de refundar la República; producto de lo cual, ese mismo año, vio la luz la Constitución de la República Bolivariana de Venezuela. Esta Carta Magna, entre otros aspectos, postula un Estado social de Derecho y de Justicia, amplía los derechos sociales y concibe a la salud como un derecho humano fundamental, parte integrante del derecho a la vida y concibe al Estado como garante y promotor de políticas orientadas a elevar la calidad de vida, el bienestar colectivo y el acceso a los servicios.

$\mathrm{Al}$ amparo de sus cartas magnas, la del 99 y las precedentes, Venezuela ha suscrito y ratificado las declaraciones, tratados y pactos internacionales más significativos en materia de Derechos Humanos y desarrollo, entre las que destacan la Declaración Universal de los Derechos Humanos, el Pacto Internacional de Derechos Económicos, Sociales y Culturales, la Convención de los Derechos del Niño, la Declaración sobre el Desarrollo y la Agenda 2030 para el desarrollo sostenible; en específico, La constitución vigente establece que los instrumentos que regulan derechos humanos tienen jerarquía constitucional y son de aplicación inmediata.

La Ley Orgánica de Protección de Niños, Niñas y Adolescentes ratifica la Doctrina integral de protección a la infancia prevista en la Convención de los Derechos del niño. También establece al Estado venezolano la "obligación indeclinable" de tomar las medidas administrativas, legislativas, judiciales, y de cualquier otra índole que sean necesarias y apropiadas para asegurar a esta población el disfrute pleno y efectivo de sus derechos.

Bajo el paraguas de la Constitución de 1999, en el área de salud, entró en funcionamiento un modelo de Atención Integral de Salud para fortalecer la red ambulatoria, encargada de la atención primaria. En sus primeros diez años de vigencia se inició un proceso de transición epidemiológica caracterizado por una disminución de las enfermedades infecciosas y un incremento de las enfermedades no transmisibles y las lesiones. En el año 2000, el Ministerio de Salud instaló el Comité Nacional de Prevención y Control de la Mortalidad Materna e Infantil y dispuso mejorar los tres niveles de atención sanitaria; no obstante; la tendencia al descenso de la mortalidad infantil se ralentizó e incluso hubo incrementos entre 2001 y 2003. La tasa osciló entre 26,17 y 22,02 (Bonvecchio et al, 2011; Correa, 2017).

Pese a los nuevos paradigmas normativos y las declaraciones, para la primera década del 2000 las tasas de mortalidad infantil en Venezuela se incrementaron sustancialmente; las cifras se asemejaban a las observadas a mediados de la década de 1990. Para 2008, la Federación Médica de Venezuela advirtió sobre un cambio del enfoque preventivo hacia el médico-curativo, expresado en la reducción de los programas de vacunación y la baja cobertura en vacunas, lo que facilitó, 
para entonces: "la reaparición de brotes epidémicos de enfermedades transmisibles, controlables mediante vacunas y el riesgo de aparición de otras con grave impacto epidémico como poliomielitis y difteria que fueron erradicadas hace muchos años" (2000, p. 144). En este mismo año, la tasa de mortalidad infantil fue de 15.8 por 1000 nacidos vivos (García et al, 2019; GIEMN, 2018; Correa, 2017).

A partir de 2010 las cifras de mortalidad infantil iniciaron un comportamiento creciente. En 2012 la tasa de mortalidad en menores de cinco años se ubicó en 17,2; en 2016, fue de 21,34 niños muertos por cada mil nacidos vivos y en 2019 las estimaciones ubican esa misma tasa en menores de cinco años en 24,2 por mil nacidos vivos. Las causas de estas muertes fueron debidas, básicamente, a afecciones del periodo perinatal, anomalías congénitas, bronquitis, infecciones intestinales y respiratorias. Y se reportan casos de morbilidad infantil debido a enfermedades ya erradicadas como la difteria, malaria, sarampión, que no se habían notificado desde comienzos de los noventa (MPPS, 2016; Bonvecchio et al, 2011; Correa, 2017).

En el año 2015, un conjunto de Organizaciones de Defensa del Derecho a la Salud venezolanas denunció ante el Consejo de Derechos Humanos de las Naciones Unidas, con motivo del Examen del 3er Informe Periódico del Estado Venezolano en el Período de Sesión 55 $5^{\circ}$ del PIDESC, del fallecimiento de 7.000 niños menores de un año, de los cuales $60 \%$ fueron recién nacidos o neonatos; así como de embarazadas y recién nacidos que habían recibido una atención adecuada y oportuna en maternidades y unidades pediátricas. Ante las denuncias documentadas, el Consejo de Derechos Humanos manifestó preocupación por la limitada efectividad de los derechos humanos de la población infantil en el país, específicamente por el estancamiento de las cifras de mortalidad infantil y destacó la inexistencia de suficientes servicios médicos y medicamentos en hospitales, y de políticas y centros de salud mental para la atención (Naciones Unidas, 2016).

A estas observaciones la República respondió que la mortalidad infantil había registrado "una importante disminución", al punto que la variación porcentual de la tasa de mortalidad en menores de un año había presentado un descenso de 31,90\% y la tasa de mortalidad infantil para 2015 se había ubicado en 14,79\%; aunque admitió que "el mejoramiento de la atención al neonato" constituía "un reto para avanzar en la disminución de la mortalidad neonatal" (Naciones Unidas, 2016, punto 50). Pese al compromiso, para la primera quincena del mes de mayo de 2016, fecha en que se publicó el último Boletín Epidemiológico del Ministerio del Poder Popular para la Salud (1), la tasa fue de 21,34 niños muertos por cada mil nacidos vivos (MPP, 2016).

(1) En el país existe una marcada opacidad en el manejo de la información pública, lo que se expresa en irregularidad o ausencia de publicación de datos. En materia de salud desde 2013 y 2014 
En enero de 2018, Unicef dio a conocer un comunicado de prensa en el que destacó que la "prevalencia de emaciación" en niños menores de cinco años aumentó de 3,2\% en el 2009 a 4,1\% en el 2017 y la desnutrición creció de un 10,5\% a un 13\% entre 2004-2006 y 2014-2016. Por su parte, la Relatoría Especial sobre Derechos Económicos, Sociales, Culturales y Ambientales de la Comisión Interamericana de Derechos Humanos reportó en 2018 que, en Venezuela, "durante 2017 habrían fallecido entre cinco y seis niños semanalmente por falta de alimentación, y al menos $33 \%$ de la población infantil presentaría indicadores de retardo en su crecimiento". Agregó igualmente que "el $11.4 \%$ de la población infantil ya se encontraría en situación de desnutrición" (OEA, 2018, p. 1).

El 26 septiembre de ese mismo año el Consejo de Derechos Humanos de las Naciones Unidas aprobó la Resolución Promoción y protección de los derechos humanos en la República Bolivariana de Venezuela en la que, entre otros razonamientos, se admitió la existencia de una crisis política, económica, social y humanitaria en Venezuela y, se exhortó a su gobierno a aceptar la asistencia humanitaria, para hacerle frente "a la escasez de alimentos, medicamentos y suministros médicos, el aumento de la malnutrición, especialmente entre los niños" (Naciones Unidas, 2018, p. 2). En ese mismo año, la Organización Panamericana de la Salud manifestó su inquietud por el aumento de la mortalidad materna y en menores de 1 año y la falta de acceso a los medicamentos en el país (OPS, 2018).

En 2019 las estimaciones ubican esa misma tasa en menores de cinco años en 24,2 por mil nacidos vivos. Las causas de estas muertes fueron debidas, básicamente, a afecciones del periodo perinatal, anomalías congénitas, bronquitis, infecciones intestinales y respiratorias. Y se reportan casos de morbilidad infantil debido a enfermedades ya erradicadas como la difteria, malaria, sarampión que no se habían notificado desde comienzos de los noventa. Ese mismo año, el Coordinador de Asuntos Humanitarios de las Naciones Unidas declaró ante el Consejo de Seguridad la existencia de un "problema humanitario muy real en Venezuela" (Lowcock, 2019, p. 1) que abarcaba a más de 7 millones de personas en el país, entre los cuales destacó la situación de los niños menores de 5 años, de las mujeres embarazadas, los lactantes y los indígenas (MPPS, 2016; Correa, 2017; García et al, 2019).

no se publican los Anuarios de Mortalidad y Morbilidad. Los boletines epidemiológicos semanales del Ministerio del Poder Popular para la Salud sobre enfermedades de notificación obligatoria, enfermedades prevenibles por vacuna, enfermedades por vectores, mortalidad materna e infantil, se interrumpieron en 2014; luego, se publicó el de la semana 52 del 25 al 31 de diciembre de 2016, sin que hasta la fecha haya habido otra. La última Memoria que hizo pública el MPPS Fue la correspondiente a 2015. 
A finales de ese año 2019 se presentó la estrategia conjunta para la planeación de la respuesta humanitaria a la situación venezolana, para mitigar significativamente el impacto humanitario de la crisis en Venezuela debido al limitado acceso a servicios médicos críticos y esenciales, al mantenimiento de los equipos médicos y líneas vitales, y al abastecimiento y distribución continua de medicamentos e insumos médicos.

Conforme a los estudios de la Cepal (2010) y de la OCDE (2020) que se han venido citando, en Venezuela la mortalidad infantil se ha venido incrementando en un $40 \%$ y, como en otros países de la región, esto tiene una estrecha relación con el nivel de ingresos y el nivel educativo de la madre; siendo el caso que el riesgo de muerte de esta población es el doble cuando esos niños pertenecen a la etnia Wayú, ubicada al Occidente del país.

El aumento de las tasas de mortalidad infantil es consistente con dos premisas. Por una parte, las acciones del Estado venezolano para disminuir las cifras de mortalidad infantil no han sido consistentes con los resultados obtenidos y, por la otra, tales resultados comprometen abiertamente su responsabilidad al no garantizar el derecho a la salud y otros derechos humanos de esta población, con lo cual hay una violación de los acuerdos internacionales suscritos por la República.

Como dijera Ban Ki Moon, antiguo secretario de la ONU: "Las violaciones de los derechos humanos son algo más que tragedias personales. Son una alarma que puede advertir de crisis mucho mayores" (Naciones Unidas, 2014, p. 1). Indicadores objetivos muestran un deterioro progresivo de los derechos humanos en Venezuela; su población, y muy especialmente la infantil, pierde aceleradamente oportunidades que hacen posible el desarrollo y, más allá sus posibilidades de vivir.

\section{A manera de cierre}

Cuando un país reconoce en su legislación interna los derechos humanos, lo que hace es darles una mayor legitimidad y aumentar los canales de exigibilidad dentro de su ordenamiento. En el caso venezolano, la Constitución de 1999 abrió un paraguas en ese sentido. Pese a esos notables avances jurídicos, el ejercicio efectivo de los derechos humanos en el país se ha venido resintiendo progresivamente en el país, en los últimos años, hasta límites que han hecho sonar las alarmas de los organismos internacionales con competencia en la materia.

Como han venido dando cuenta diversos actores gubernamentales y no gubernamentales, nacionales e internacionales, la garantía y ejercicio del derecho a la salud se ha venido limitando, en los hechos. El estado y funcionamiento de la infraestructura sanitaria, de los equipos médico-quirúrgicos, los servicios de salud, 
el tipo, calidad y oportunidad de los tratamientos y su dispensa visibilizan, en su conjunto, serios desafíos para la comunidad nacional e internacional.

Un indicador muy sensible para evaluar la situación y el bienestar de la población de un país de un país es la mortalidad infantil. Investigaciones mundiales, regionales y nacionales que abordan este tema, a partir de las estadísticas con que se cuenta, señalan que la mortalidad infantil ha disminuido a nivel global, sobre todo en los países más desarrollados, y que tal disminución obedece a cambios sustantivos en la realidad socio económica y progresos sanitarios, a la par de un acceso oportuno a servicios sanitarios de calidad.

En el caso venezolano ha ocurrido lo contrario, las tendencias hacia la disminución que experimentaron las tasas de mortalidad infantil desde mediados del siglo XX, se han venido revirtiendo al punto que hoy son motivo de inquietud para especialistas, organizaciones no gubernamentales y organismos internacionales. Los documentos revisados muestran que, desde finales del siglo pasado hasta la actualidad, la mortalidad infantil se ha reducido en casi un $70 \%$ en el mundo; mientras que en Venezuela ha aumentado, con lo significa el riesgo en el que vive esa población y el incumplimiento por parte del Estado de su obligación de respetar, proteger y garantizarles sus derechos humanos.

La creación de condiciones para mejorar la calidad de vida de toda población, la situación sanitaria del país, y mejorar el acceso a los servicios, establecimientos y programas de salud de calidad es un imperativo; lo que requiere de políticas públicas con potencial impacto en la salud y desarrollo de las personas. Las cifras de fallecimientos de niños por causas evitables y prevenibles es una deuda social que aumenta cada día y demanda esfuerzos para reestablecer las garantías efectivas de sus derechos humanos, muy especialmente a la vida, la salud y a la no discriminación.

\section{Bibliografía}

Abriata, M. G. y Fandiño. E. (2010). Abordaje de la mortalidad infantil en Argentina desde la perspectiva de las desigualdades en salud. Revista Argentina de Salud Pública, vol. 1, No 2, Marzo (pp. 43-45). Recuperado de https://ojsrasp.msal.gov. ar/index.php/rasp/article/view/452/382

Aguirre, A. (2009). La mortalidad infantil y la mortalidad materna en el siglo XX. Papeles de Población, №. 61, julio/septiembre (pp. 75-99). Recuperado de https:// www.redalyc.org/pdf/112/11211806005.pdf

Augsburger, A.; Galende, S.; Gerlero, S. y Moyano, C. (2006). Mortalidad infantil y condiciones de vida La producción de información epidemiológica como aporte 
al proceso político de descentralización municipal en Rosario (Argentina). Kairos, año 10, No 18. Recuperado de http://www.revistakairos.org

Banco Mundial (2015). Objetivos de desarrollo del milenio. Recuperado de http://www5.bancomundial.org/odm/muerte-infantil.html

Bolsi, A.; Longhi, F. y Paolasso, P. (2009). Pobreza y mortalidad infantil en el Norte Grande Argentino. Un aporte para la formulación de políticas públicas. Cuadernos Geográficos, 45 (2009-2) (pp. 231-261). Recuperado de https://revistaseug.ugr.es/index.php/cuadgeo/article/view/765

Bonvecchio, A.; Becerril-Montekio, V.; Carriedo-Lutzenkirchen, Á. y Landaeta-Jiménez, M. (2011). Sistema de salud de Venezuela. Salud pública de México, vol. 53, suplemento 2 de 2011 (pp. 274-286). Recuperado de http://www.scielo. org.mx/scielo.php?script=sci_arttext\&pid=S0036-

Castellanos, P. L. (1982). Notas sobre el Estado y la salud en Venezuela. Cuadernos de la sociedad venezolana de planificación, №156 -158 (pp. 69 -152). Caracas.

Cepal (2010). Mortalidad infantil y en la niñez de pueblos indígenas y afrodescendientes de América Latina: inequidades estructurales, patrones diversos y evidencia de derechos no cumplidos, documento de proyecto, volumen 6 de la serie "Enfoque étnico en las fuentes de datos y estadísticas de salud". Recuperado de https://www.cepal.org/sites/default/files/publication/files/3798/lcw348.pdf

Cepal y Unicef (2011). Mortalidad en la niñez. Una base de datos de América Latina desde 1960. Recuperado de https://www.google.com/search?q=Mortal idad+en+la+ni\%C3\%Blez.+Una+base+de+datos+de++Am\%C3\%A9rica+Latin $\mathrm{a}+$ desde $+1960 \&$ safe $=$ active $\&$ sxsrf $=$ ALeKk01K0PoJhHQmkCm4vHgX7BE3RY6a rg\%3A1618925497355\&ei=udd-

Correa, G. (2017). Mortalidad infantil en el contexto de la crisis venezolana actual. En Anitza Freites (coord.), Venezuela a caída sin fin ¿hasta cuándo? Encuesta Nacional de Condiciones de Vida 2016 (ENCOVI) (pp. 151-169). Venezuela: Ucab.

Dhrifi, A. (2018). Gastos en salud, crecimiento económico y mortalidad infantil: antecedentes de países desarrollados y en desarrollo. Revista de la CEPAL, № 125, agosto. Recuperado de https://www.cepal.org/es/publicaciones/43992-gastossalud-crecimiento-economico-mortalidad-infantil-antecedentes-paises

Díaz, Y. (2003). ¿Es necesario sacrificar equidad para alcanzar Desarrollo?: el caso de las inequidades en Mortalidad infantil en Colombia. Documento CEDE 2003-11, abril. Recuperado de https://ideas.repec.org/p/col/000089/003804.html 
LA MORTALIDAD INFANTIL COMO PROBLEMA DE DERECHOS HUMANOS: EL CASO VENEZOLANO

- ANDy Delgado Blanco (PP. 325-346)

Federación Médica de Venezuela (2008). Diagnóstico del sector salud en Venezuela: estudios de las enfermedades emergentes y reemergentes. Ponencia central presentada en la LXIII Reunión Ordinaria de la Asamblea, realizada en Punto Fijo, estado Falcón, del 27 al 31 de octubre (p. 144).

García, J.; Correa G. y Rousset, B. (2019). Trends in infant mortality in Venezuela between 1985 and 2016: a systematic analysis of demographic data. The Lancet. Vol. 7, issue 3. March 01, 2019 (pp. 331-336). Recuperado de https://www.thelancet.com/journals/langlo/article/PIIS2214-109X(18)30479-0/fulltext

Grupo Interinstitucional para la Estimación de la Mortalidad en la Niñez (GIEMN) (2018). Informe 2018. Recuperado de https://scalingupnutrition.org/es/ news/indices-y-tendencias-en-mortalidad-infantil-informe-de-2018/; https:// childmortality.org/data/Venezuela\%20(Bolivarian\%20Republic\%20of)

Lowcock, M. (2019). Asistencia humanitaria en Venezuela. Recuperado de https://www.aa.com.tr/es/mundo/experto-de-onu-afirma-que-en-venezuelase-deben-separar-objetivos-pol\%C3\%ADticos-y-humanitarios/1448473

Ministerio del Poder Popular para la Salud (2016). Boletín Epidemiológico. Recuperado de http://www.mppp.gob.ve/wp-content.

Medina Gómez, O. y López Arellano, O. (2011). Asociación de los tipos de carencia y grado de desarrollo humano con la mortalidad infantil en México, 2008. Cad. Saúde Pública, 27(8) (pp. 1603-1610). Rio de Janeiro Recuperado de https:// www.scielosp.org/article/csp/2011.v27n8/1603-1610/

Naciones Unidas. Consejo de Derechos Humanos (2018). Resolución Promoción y protección de los derechos humanos en la República Bolivariana de Venezuela. Recuperado de https://reliefweb.int/report/venezuela-bolivarian-republic/ promoci-n-y-protecci-n-de-los-derechos-humanos-en-la-rep-blica

Naciones Unidas. Consejo de Derechos Humanos. Grupo de Trabajo sobre el Examen Periódico Universal (2016). Informe nacional presentado con arreglo al párrafo 5 del anexo de la resolución 16/21 del Consejo de Derechos Humanos. República de Venezuela. $26^{\circ}$ período de sesiones. 31 de octubre a 11 de noviembre de 2016. Recuperado de https://digitallibrary.un.org/record/761971?ln=es

Naciones Unidas (2014). Memoria del Secretario General sobre la labor de la Organización. Recuperado de http://www.un.org/sg/rightsupfront/

Naciones Unidas. Comité de derechos económicos, sociales y culturales. (2000). Cuestiones sustantivas que se plantean en la aplicación del Pacto internacional de derechos económicos, sociales y culturales. El derecho al disfrute del más alto nivel 
posible de salud. Observación General 14. Recuperado de http://www.acnur.org/ fileadmin/Documentos

Naciones Unidades (1994). Programa de Acción aprobado en la Conferencia Internacional sobre la Población y el Desarrollo. El Cairo, septiembre de 1994. Recuperado de https://www.unfpa.org/sites/default/files/pub-pdf/ICPD-PoA-EsFINAL.pdf

Naciones Unidas (1990). Declaración mundial sobre la supervivencia, la protección y el desarrollo del niño. Cumbre Mundial a favor de la infancia. Nueva York, 1990. Recuperado de http://www.unicef.org/wsc/declare.htm

Naciones Unidas (1948). Declaración Universal de los Derechos Humanos. Recuperado de https://www.un.org/es/about-us/universal-declaration-of-human-

OCDE (2020). Panorama de la Salud: Latinoamérica y el Caribe 2020. Recuperado de https://www.oecd-ilibrary.org/sites/2b388fe2-es/index.html?itemId=/ content/component/2b388fe2-es\#indicator-dle16578

Orbea López, M. (2019). La mortalidad en la niñez, un tema de prioridad a nivel internacional. CEDEM / Novedades en población, No 30, julio-diciembre (pp. 178-188). Recuperado de http://www.novpob.uh.cu

Organización de Estados Americanos (2018). CIDH y su Relatoría Especial sobre Derechos Económicos, Sociales, Culturales y Ambientales. Recuperado de http:// www.oas.org/es/cidh/prensa/comunicados/2018/016.asp

Organización Panamericana de la Salud (2001). Desigualdades en la Mortalidad Infantil en la Región de las Américas: Elementos Básicos para el Análisis. Bol. Epidem. para América Latina. diciembre 2001, 22(2) (pp. 4-7). Recuperado de https://www.paho.org/spanish/sha/be_v22n2-DesigualdadesIM.htm

Organización Panamericana de la Salud (2018). Respuesta de la OPS para mantener una Agenda eficaz de cooperación técnica en Venezuela y en los estados miembros vecinos. Washington D.C, $56^{\text {a }}$ Sesión Consejo Directivo. Recuperado de https://iris.paho.org/handle/10665.2/53028 y https://www.iapo.org.uk/sites/default/files/files/IAPO\%20PAHO_Punto\%208_12\%20de\%20la\%20Agenda\%20Respuesta\%20de\%20la\%20OPS\%20para\%20mantener\%20una\%20agenda\%20de\%20 cooperaci\%C3\%B3n\%20t\%C3\%A9cnica\%20en\%20Venezuela\%20y\%20en\%20 los\%20Estados\%20Miembros\%20vecinos.pdf

Organización Mundial de la Salud (2014). Comunicado de prensa conjunto OMS, UNICEF, Grupo del Banco Mundial. Datos recientes revelan un rápido descenso sin precedentes en las tasas de mortalidad infantil. Recuperado de https://www.paho. 
org/hq/index.php?option=com_content\&view=article\&id=9956:2014-new-datashow-child-mortality-rates-falling-faster-than-ever\&Itemid=135\&lang=es

Organización Mundial de la Salud (2020). Mejorar la supervivencia y el bienestar de los niños, Recuperado de https://www.who.int/es/news-room/fact-sheets/ detail/children-reducing-mortality\#: :text=Desde\%201990\%20se\%20han\%20 logrado,5\%2C2\%20millones\%20en\%202019

Páez S. G. (2018). Venezuela: de una mortalidad en descenso a una en ascenso, 1950-2012 / 2017-2020. Revista Geográfica Venezolana, vol. 51 (1), enero-junio (pp. 70-87). Venezuela: Universidad de los Andes. Recuperado de https://www. redalyc.org/jatsRepo/3477/347760307006/html/index.html

Pison, G. (2010). Le recul de la mortalité des enfants dans le monde: de grandes inégalités entre pays. Population \& Sociétés no 463, janvier 2010, Bulletin mensuel d'information de l'Institut national d'études démographiques (pp. 1-4). Recuperado de https://www.ined.fr/fr/publications/editions/population-et-societes/lerecul-de-la-mortalite-des-enfants-dans-le-monde-de-grandes-inegalites-entrepays/

Sen, A. (2002). El derecho a no tener hambre. Colombia: Universidad Externado de Colombia. Centro de Investigación en Filosofía y Derecho.

Sen, A. (2000). Desarrollo y libertad. Barcelona: Editorial Planeta.

UNICEF (2018). Niños y niñas en América Latina y El Caribe. Panorama 2018. Recuperado de https://www.unicef.org/lac/informes/ninos-y-ninas-en-americalatina-y-el-caribe

Yánez Magallanes, C. (2013). Mortalidad infantil: comportamiento epidemiológico. Trabajo especial de grado que se presenta para optar al título de especialista en Epidemiología. Facultad de Medicina, Escuela de salud pública. Especialización en epidemiologia. Universidad central de Venezuela.

\section{Páginas web}

Mortalidad Infantil(s/f). Recuperado de https://conceptodefinicion.de/mortalidad-infantil/

Fecha de recepción: 31-03-2021 Fecha de aceptación: 08-09-2021 
